\title{
LHC COLLIMATION SYSTEM HARDWARE COMMISSIONING
}

\author{
Th. Weiler, O. Aberle, R. Assmann, R. Chamizo, Y. Kadi, J. Lettry, R. Losito, S. Redaelli \\ CERN, Geneva, Switzerland
}

\begin{abstract}
The stored energy and intensity of the LHC beam exceed the damage level of the machine and the quench level of the magnets by far. Therefore a robust and reliable collimation system is required which prevents the quenching of the magnets during regular operation and protects the accelerator components from damage in the event of beam loss. To assure that the installed collimators will protect the machine and permit the required performance of the collider, an appropriate hardware commissioning has to be implemented. In this contribution we describe the procedures for the hardware commissioning of the LHC collimation system. These procedures will establish the required precision and reliability of collimator movements and settings before the start of beam operation.
\end{abstract}

CERN, 


\title{
LHC Collimation System Hardware Commissioning
}

\author{
Th. Weiler*, O. Aberle, R. Assmann, R. Chamizo, Y. Kadi, J. Lettry, R. Losito, S. Redaelli, \\ CERN, Geneva, Switzerland
}

\begin{abstract}
The stored energy and intensity of the LHC beam exceed the damage level of the machine and the quench level of the magnets by far. Therefore a robust and reliable collimation system is required which prevents the quenching of the magnets during regular operation and protects the accelerator components from damage in the event of beam loss. To assure that the installed collimators will protect the machine and permit the required performance of the collider, an appropriate hardware commissioning has to be implemented. In this contribution we describe the procedures for the hardware commissioning of the LHC collimation system. These procedures will establish the required precision and reliability of collimator movements and settings before the start of beam operation.
\end{abstract}

\section{INTRODUCTION}

At the Large Hadron Collider (LHC) two proton beams with a centre of mass energy of $14 \mathrm{TeV}$ are stored. At design luminosity each beam consists of 2808 bunches containing each $1.15 \times 10^{11}$ protons [1]. The stored energy and intensity of the beam exceeds the damage level of the machine and quench level of the magnets by far. A robust and reliable collimation system for quench and machine protection is needed.

The task to efficiently clean the beam halo, which is continuously filled by beam dynamics processes, and protect the machine from accidents, requires a highly robust and reliable design [2]. Also the requirements on the mechanical precision are demanding, considering the typical beam size of $200 \mu \mathrm{m}$, a surface flatness of the jaw along beam direction that has to be below $40 \mu \mathrm{m}$ and the accuracy of the jaw motion that has to reach $10 \mu \mathrm{m}$.

In this paper the LHC-collimator design is briefly reviewed and the steps prepared for the hardware commissioning are summarised.

\section{COLLIMATOR DESIGN}

The technical details of the design, like choice of material, can be found in $[2,3]$. Here only a coarse overview of the collimator and supports is given.

The fully installed collimator consists of five main mechanical parts: (1) the base-support providing the stand of the collimator in the tunnel. (2) the alignment plate, allowing the adjustment of the collimator, (3) the lower plugin, providing cooling water and electrical connection to the

\footnotetext{
* Thomas.Weiler@cern.ch
}

collimator. These three parts compose the base-support assembly. Figure 1 shows a base-support assembly as installed in the LHC tunnel.

(4) the main part of the collimator is the vacuum tank comprising the two jaws and hosting the tables and motors for moving the jaws. Figure 2 shows a view along the jaws of a series TCS-type collimator before closing the vacuum tank. The vacuum tank will be mounted to an uppersupport (5). This composition is called upper-support assembly and will host all needed parts for the installation in the tunnel on a base-support assembly. Figure 3 shows a TCS-type collimator installed in the LHC-tunnel.

The separation into base-support assembly and uppersupport assembly allows a quick installation and also a quick exchange of a collimator in case of damage. This is required due to the high activation in cleaning insertions.

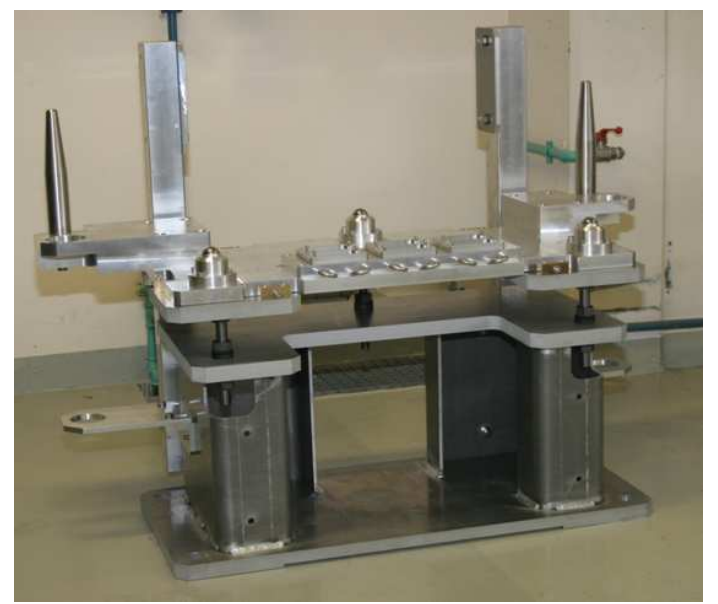

Figure 1: Photograph of an collimator base-support assembly, including lower plug-in and alignment plate.

\section{HARDWARE COMMISSIONING}

The hardware commissioning of the LHC collimation system is divided into several steps [4]. Figure 4 summarises the production, final assembly and hardware commissioning of the LHC collimation system. Hardware commissioning already starts during final assembly with calibration and alignment procedures assuring that only fully conform collimators are installed in the tunnel. Parameters tested and the relevant tolerances include: surface flatness $(40 \mu \mathrm{m})$, torque measurement $(0.5 \mathrm{Nm})$, vacuum pressure $\left(<4 \times 10^{-8} \mathrm{mbar}\right)$, mechanical play $(<20 \mu \mathrm{m})$, minimum gap $(<5 \mathrm{~mm})$, maximum jaw tilt angle $(>2 \mu \mathrm{rad})$ and minimum jaw tilt angle $(<2 \mu \mathrm{rad})$. 


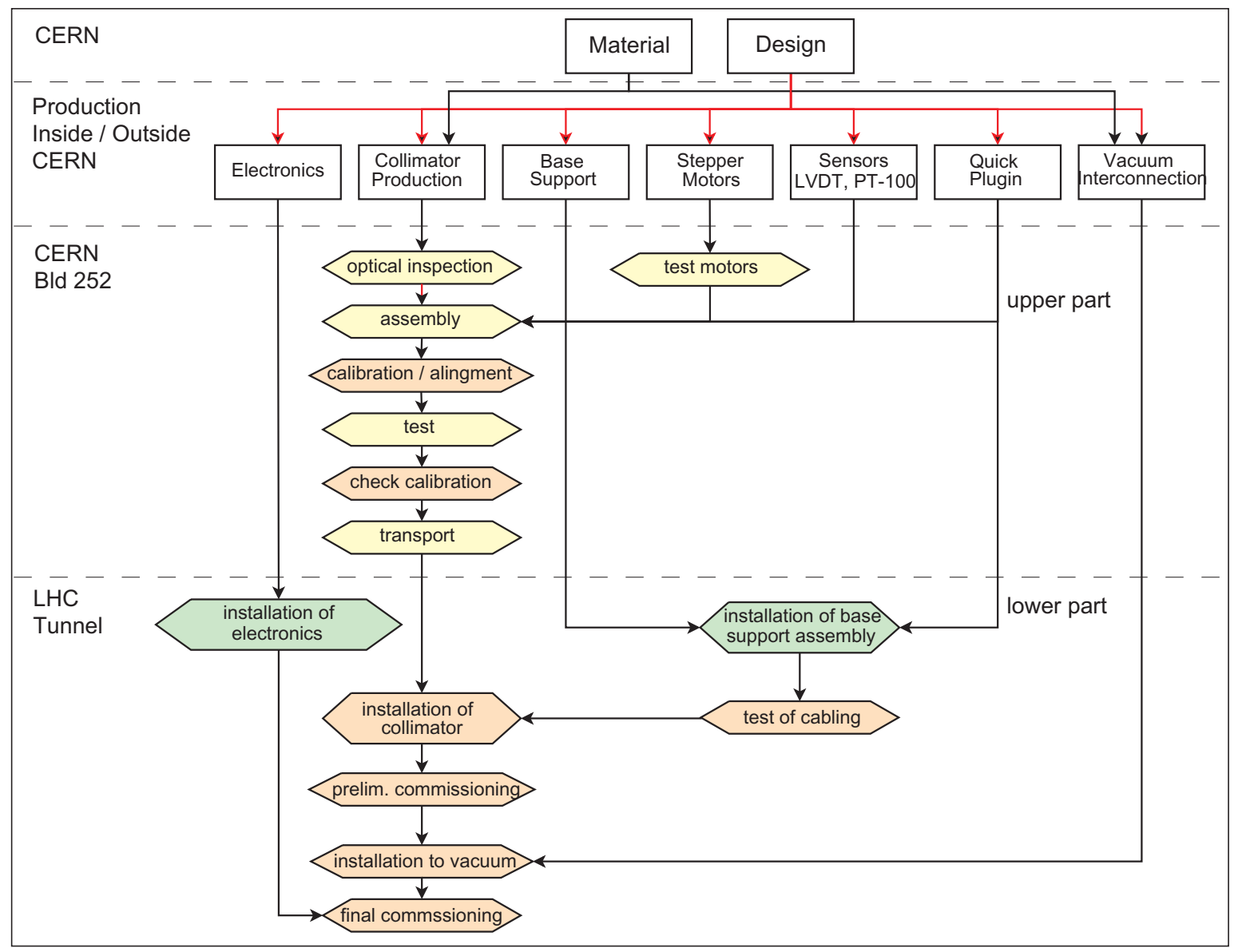

Figure 4: This flowchart summarises the production, final assembly and hardware commissioning of the LHC collimation system. The hardware commissioning starts already during assembly with calibration and alignment procedures assuring that only fully conform collimators are installed in the tunnel. The boxes with orange colour involve steps on hardware commissioning.

\section{Test on Tunnel Infrastructure}

Test on the tunnel infrastructure are performed prior to installation of the collimator. The leak tightness of the cooling water supply up to the lower plug-in is established and verified if the adjustment of the flow rate by valves is applicable. In addition, the cabling from the rack location up to the lower plug-in is checked. This test includes the test of the cable response, the resistivity and the correct pin assignment and labelling.

\section{Test on Tunnel Equipment}

After the collimator is installed in the tunnel, the water tightness of the system up to the collimator will be checked by applying the nominal cooling to the circuit. The flow will be adjusted to the specified value $(5 \mathrm{l} / \mathrm{min})$. All following tests will be done with cooling water applied. Thereafter movability of the collimator is tested,good readings for the position and temperature sensors are checked and limiting switches are verified.

\section{Full System Test and Calibration}

After the collimator functionality was verified, the position sensor have to be calibrated to an accuracy of $20 \mu \mathrm{m}$, using the final electronics and cables. The communication with the collimation supervisory system and low level system is verified, allowing further testing of the system with the control room software. After these test have been done, the interlock chain can be tested. All possible interlock conditions (e.g. jaw temperature limit reached) are generated by the collimator and sent to the machine interlock system to verify the proper handling of the signals.

\section{Test on Installed Collimator Under Vacuum}

So far all tests on the collimator in the tunnel were conducted under normal pressure, no vacuum established in the vacuum tank of the collimator, after it left the surface laboratory. When the vacuum is applied the forces on the mechanics may change. Therefore the basic functionality will be cross-checked once the vacuum is established and final mechanical forces are in place. The calibration of the position sensor are validated under vacuum, gain and offset 


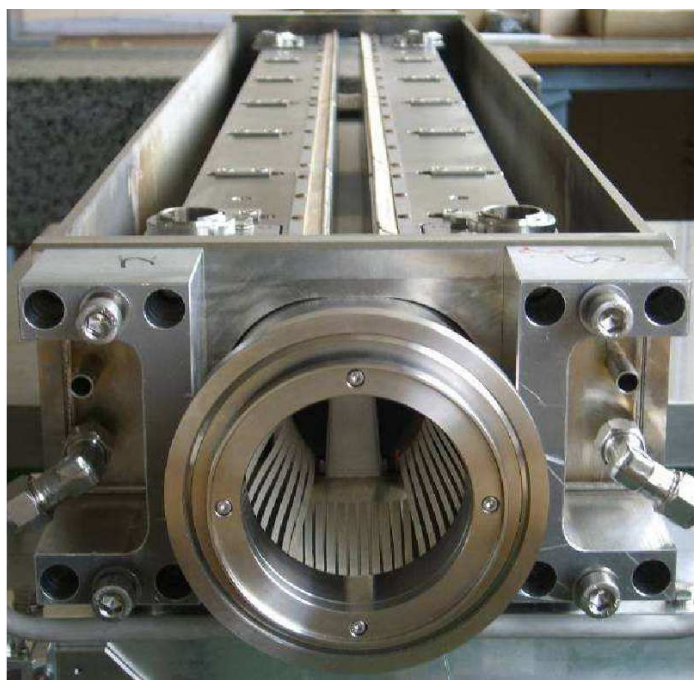

Figure 2: View along the collimator-jaws of a series TCStype collimator before closing of vacuum tank.

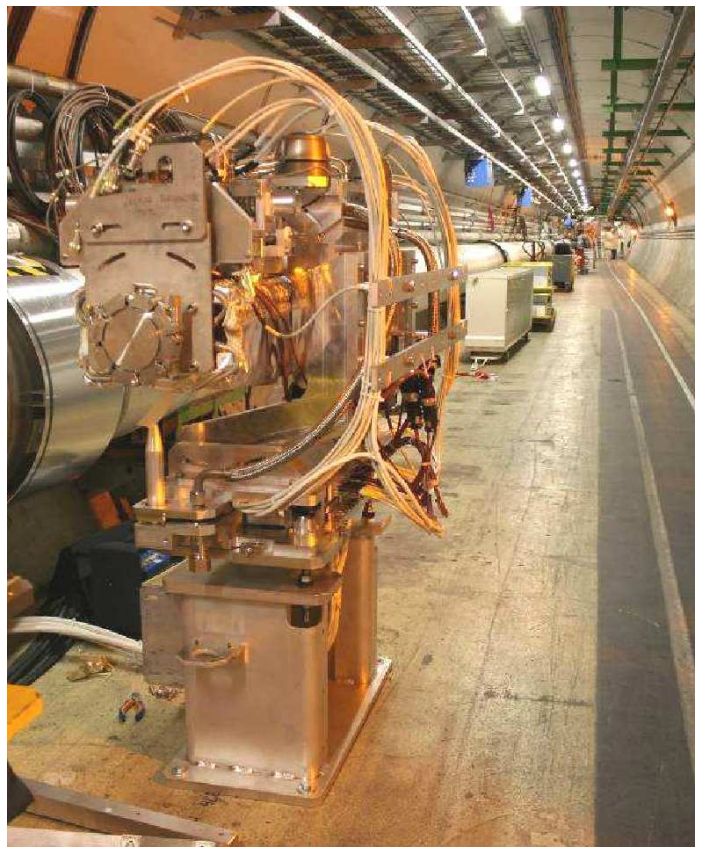

Figure 3: Photograph of LHC collimator installed on its base-support assembly (base-support and quick plug-in).

of the sensors are adjusted if needed. A further important measurement is the mechanical play, which can be easily accessed by moving the collimator jaw in small steps and reverting the direction of the movement. The resulting mechanical play can be measured with an accuracy of $5 \mu \mathrm{m}$. Repeating this measurement during the LHC operation can give hints on an abrasion or radiation damage of the mechanics.

\section{MTF implementation}

To keep track on the hardware commissioning and collect all measured data in a central place, the hardware commissioning steps for all collimators are implemented in the Manufacturing and Test Folder (MTF) database. The hardware commissioning data will be linked to the production and assembly data to give full access to all available data on the collimator and its production history. Figure 5 shows as an example the available data on MTF for the minimum gap on the end switch for the first collimators hardware commissioned in the surface laboratory.

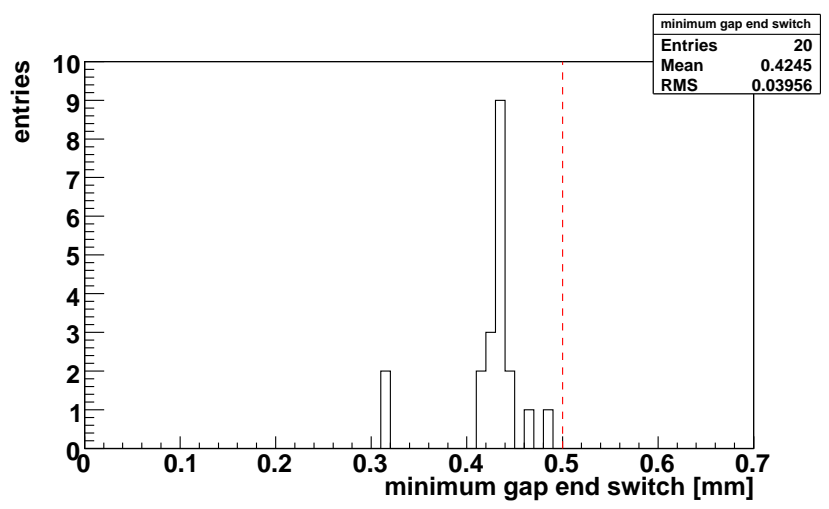

Figure 5: Minimum gap on end switch for the first collimators hardware commissioned in the surface laboratory. The dashed line shows the specified limit of $0.5 \mathrm{~mm}$.

\section{CONCLUSION}

The hardware commissioning procedures presented here should allow a smooth installation and testing of the collimation system according to the schedule of the LHC. All essential parameters of the system will be measured and made accessible through a database system. This is useful for continuous monitoring of the system parameters and identification of possible weak points in the material.

\section{REFERENCES}

[1] O. Brüning et al.,'LHC Design Report, Vol.1 The LHC Main Ring ",CERN-2004-003.

[2] R. Assmann et al.,'The Final Collimation System for the LHC', Proceedings of European Particle Accelerator Conference, Edinburgh 2006.

[3] A. Bertarelli, et al., "The Mechanical Design for the LHC Colliators", Proceedings of European PArticle Accelarator Conference, Lucerne 2004.

[4] Th. Weiler et al., "Collimator Final Assembly and Hardware Commissioning for LHC”, LHC Project Document, in preparation, 2007 